\title{
Capsule Commentary on Michaelidis et al., Cost-Effectiveness of Decision Support Strategies in Acute Bronchitis
}

\author{
Jeanne T. Black, Ph.D., M.B.A. \\ Cedars-Sinai Health System, Los Angeles, CA, USA. \\ J Gen Intern Med 31(3):328 \\ DOI: $10.1007 / \mathrm{s} 11606-015-3431-2$ \\ (c) Society of General Internal Medicine 2015
}

$\mathrm{U}$ sing cost-effectiveness analysis (CEA) to make decisions about health care resources faces significant political and popular opposition in the U.S. The Affordable Care Act (ACA) explicitly prohibits the use of Quality Adjusted Life Years in determining coverage or reimbursement or recommending treatments, ${ }^{1}$ and the executive director of the Patient Centered Outcomes Research Institute (PCORI) has distanced the organization from CEA altogether. ${ }^{2}$

Michaelidis, et al.'s cost-effectiveness analysis ${ }^{3}$ was based on estimates of effect derived from a cluster-randomized trial by Gonzales et al. ${ }^{4}$ that compared printed decision support (PDS) and computer-assisted decision support (CDS) with usual care. That article's point estimates for the odds ratios of antibiotic prescribing were used in the base case ( 0.57 for the PDS strategy and 0.64 for CDS). However, the $95 \%$ confidence intervals for the two strategies overlapped almost completely, indicating that the adjusted odds of antibiotic use were not different. The authors attempt to address this issue in a secondary sensitivity analysis and conclude that the PDS strategy cost \$220 less per patient. Operationally, the only difference between the two interventions was that one had posters, while the other incurred computer programming costs. The cost difference seems to have resulted because the CEA also retained the point estimates from the original trial for rates of return to clinic within 30 days and use of chest x-rays. In both cases, the nominal rates were higher at the CDS sites than at the PDS sites. Yet the original study states "Return visit rates...increased modestly at all study sites and were not significantly different among study sites." With no difference in prescriptions avoided and no difference in associated utilization, the cost difference comes down to the comparison between the direct costs of poster printing vs. computer programming. With Meaningful Use incentives driving health systems to adopt electronic decision support, it is useful to be reminded that more traditional means of physician education can be equally cost-effective. However, some would argue that a methodologically complex analysis like CEA was not required to determine that posters are probably less costly than computer programming time.

Conflict of Interest: The author has no conflicts with this article.

Corresponding Author: Jeanne T. Black, Ph.D., M.B.A.; CedarsSinai Health System, Los Angeles, CA 90048, USA (e-mail:jeanne.black@cshs.org).

\section{REFERENCES}

1. Neumann PJ, Weinstein MC. Legislating against use of cost-effectiveness information. New Engl J Med. 2010;363(16):1495-7.

2. Selby JV. October 28, 2014. http://www.pcori.org/blog/bringing-stakeholders-together-talk-about-hepatitis-c. [Accessed May 7, 2015].

3. Michaelidis CI, Kern MS, Smith KJ. Cost-effectiveness of decision support strategies in acute bronchitis. J Gen Intern Med. 2015. doi:10.1007/ s11606-015-3289-3.

4. Gonzales R, Anderer T, McCulloch CE, et al. A cluster randomized trial of decision support strategies for reducing antibiotic use in acute bronchitis. JAMA Intern Med. 2013;173(4):267-73.

This comment refers to the article available at: $h t t p: / / d x$.doi.org/10.1007/ s11606-015-3289-3.

Published online June 13, 2015 